\title{
Disponibilidade e custo de produtos para higiene bucal comercializados em um município paranense
}

\author{
Availability and cost of oral hygiene products commercialized in a city of Paraná State, Brazil
}

\section{Fábio Luiz Mialhe ${ }^{1}$, Renato Pereira Silva ${ }^{2}$, Edo Hirata ${ }^{3}$, Luciano Falcade Santos ${ }^{4}$}

\section{Resumo}

O objetivo do presente estudo foi avaliar a disponibilidade e o custo de produtos para higiene bucal, dentre eles, dentifrícios, escovas e fios/fitas dentais, em uma amostra de supermercados localizados no município de Cascavel, PR. Foram visitados doze grandes supermercados, pertencentes a diferentes redes, localizados em vários bairros da cidade, no período de 2004 a 2006. Os dados relativos à disponibilidade de diferentes marcas, tipos e preços de cada produto foram anotados em ficha clínica específica. Os dados foram analisados por meio de estatística descritiva. A disponibilidade média de escovas de dente, dentifrícios e fio/fita dental nas lojas do centro e outros bairros foi de 20/22, 39/37 e 45/34, em 2004, de 41/44, 50/47 e 48/47, em 2005, e de 7/7, 12/11 e 11/9, em 2006. É importante que os profissionais da área odontológica avaliem esses aspectos quando recomendarem a aquisição desses produtos para indivíduos com diferentes níveis socioeconômicos, a fim de promover a universalização de práticas preventivas em saúde bucal.

Palavras-chave: Higiene bucal, Dispositivos para o cuidado bucal domiciliar, Saúde bucal

\begin{abstract}
The aim of the present study was to evaluate the availability and cost of products for oral hygiene, such as toothpastes, dental brushes and dental floss in a sample of supermarkets located in different localities at the city of Cascavel, PR. Twelve great supermarkets were visited in the period between 2004 and 2006, belonging to different districts located in several areas of the city. The data of different marks and types of the products searched in each establishment and the price of each product was registered in a specific record developed by the researcher. The data were analyzed by descriptive statistics. The average availability of dental brushes, toothpastes and dental floss in establishments of downtown/districts was 20/22, 39/37 and 45/34, in 2004, 41/44, 50/47 and 48/47, in 2005, and 7/7,12/11 and 11/9, in 2006. It was verified a great variability in costs and availability of the products, which also varied in function of location of the supermarket and year of evaluation. It is important that dental professionals evaluate those aspects when recommending the acquisition of those products to patient with different socioeconomics in order to promote the universality of preventive practices in oral health.
\end{abstract}

Keywords: Oral hygiene, Dental devices, Oral health
${ }_{1}$ Professor Livre-Docente do Departamento de Odontologia Social da Faculdade de Odontologia de Piracicaba, FOP/UNICAMP.

${ }^{2}$ Doutor em Saúde Coletiva pela Faculdade de Odontologia de Piracicaba, FOP/UNICAMP.

${ }^{3}$ Professor Doutor da Área de Odontopediatria da Universidade Estadual do Oeste do Paraná, UNIOESTE, campus Cascavel.

${ }^{4}$ Cirurgião-Dentista

\section{Introdução}

O biofilme dental, associado a fatores determinantes e modificadores, é considerado importante agente etiológico para o desenvolvimento das principais doenças bucais, tais como a cárie dentária, a gengivite e a periodontite (AXELSSON; LINDHE, 1981; ALBANDAR, 2002).

A literatura evidencia com clareza que, a escovação dental e outros procedimentos de higienização mecânicos, caso realizados de forma satisfatória e a intervalos regulares, podem controlar o biofilme bacterian com eficiência (VAN DER WEIJDEN; HIOE, 2005).

Apesar de existir uma imensa variedade de tipos de escovas dentais no mercado, há ainda insuficiente evidência de que um determinado desenho seja superior a outro. Atualmente, as escovas são desenhadas com múltiplos tufos de cerdas e em diferentes padrões, a fim de melhorar a remoção do biofilme em áreas de difícil acesso, como as faces proximais dos dentes (VAN DER WEIJDEN; HIOE, 2005).

Entretanto, as escovas dentais não conseguem remover com eficiência o biofilme localizado em todas as faces dentais. Nas faces interproximais, a remoção do biofilme é crucial, pois a maioria das doenças dentais e periodontais originam-se nestas áreas (PERRY, 2004). Apenas o uso regular do fio ou da fita dental é capaz de remover o biofilme localizado entre as faces interproximais e controlar o desenvolvimento do cálculo dentário nessas regiões (BAUROTH et al., 2003). Assim, a American Dental Association (2006) recomenda o uso diário do fio dental em adição à escovação dos dentes.

Além da escova e o fio dental, os dentifrícios atualmente exercem importante função detergente e abrasiva, sendo importantes meios de veiculação de fluoretos e outros agentes quimioterápicos (CURY, 2002).

Apesar de serem considerados os métodos mais eficientes para a remoção e o controle do biofilme, pouco estudos avaliaram a disponibilidade e os valores dos produtos supracitados em um mesmo município (CARVALHO et al., 1987; TODESCAN, 1991). Visto que muitos consumidores, principalmente os de menor renda, escolhem o local onde efetuam suas compras pela proximidade do mesmo em relação às suas residências (O Estado de São Paulo, 2010), é importante que o profissional avalie esses aspectos quando da indicação de produtos de higiene bucal para a população.

Considerando o exposto, o objetivo deste trabalho foi avaliar a disponibilidade e os valores dos produtos para higiene bucal em uma amostra de supermercados localizados em diferentes regiões do município de Cascavel, estado do Paraná, complementando a apresentação e discussão dos dados da pesquisa realizada pelos autores (SANTOS et al., 2008). 


\section{Materiais e Métodos}

Este é um estudo observacional do tipo longitudinal, visto que os dados foram coletados entre os anos de 2004 a 2006, sempre nos meses de setembro, época que a pesquisa teve início.

Para a seleção da amostra, utilizou-se a estratégia de amostragem por conglomerados, sendo que, num primeiro estágio, foram selecionadas as quatro maiores redes de supermercados do município de Cascavel, PR, e, num segundo estágio, procedeu-se a amostragem aleatória, visando a contemplar $75 \%$ dos estabelecimentos pertencentes a cada rede. Desta forma, a amostra final foi constituída por 12 lojas, localizadas em diferentes regiões do município.

Para a coleta dos dados, o pesquisador principal dirigiu-se aos locais previamente definidos e coletou os dados referentes a disponibilidade, aos valores e as especificações de escovas dentais, dentifrícios e fio/fita dental comercializados em cada estabelecimento, utilizando, para isso, ficha específica desenvolvida para o presente estudo.

Os dados foram coletados e tabulados anualmente em planilha Excel e analisados por meio de estatística descritiva. Para melhor compreensão, a análise foi inicialmente realizada por loja e, posteriormente, os dados foram analisados conjuntamente.

\section{Resultados}

Quanto à disponibilidade das escovas dentais, encontrouse um total de 11 marcas e 100 tipos de escovas dentais. No ano de 2004, foram encontrados 49 tipos, passando para 80 em 2005 e 82 em 2006. Verificou-se que 18 diferentes tipos de escovas foram retirados dos supermercados no período da pesquisa.

Ainda em relação às escovas dentais, pode-se observar através da Tabela 01 que, tratando-se da disponibilidade destes produtos por bairro, em 2004, a população que consumia em estabelicimentos próximo ao centro da cidade tinha acesso, em média, a 20 tipos de escovas por loja, e, por outro lado, os que residiam nos bairros mais periféricos apresentavam à sua disposição, em média, 22 tipos. Em 2005 ocorreu uma inversão, sendo encontrada uma média de 39 tipos de escovas no centro e 37 em outros bairros. Em 2006, os estabelecimentos localizados na região central apresentaram uma diferença positiva de aproximadamente 12 produtos, sendo que a média de tipos de escovas dentais foi de 45 nas lojas do centro contra 34 nas dos outros bairros.

Em relação à disponibilidade dos dentifrícios, encontrouse um total de 19 marcas e 95 tipos. Em 2004, o total foi de 58 tipos, passando para 82 em 2005 e 81 em 2006. Entretanto, 14 diferentes tipos foram retirados dos mercados e não encontrados em 2006. No ano de 2004, a população que consumia nos estabelecimentos localizados na área central possuía acesso, em média, a 41 tipos de produtos por loja, já nas lojas situadas nos bairros, a média foi de 44 .
Em 2005 ocorreu uma inversão na disponibilidade com uma média de 50 no centro e 47 nos bairros. Em 2006, as lojas da região central possuíam uma diferença positiva de apenas 1 produto, sendo sua média por loja de 48 contra 47 em outros bairros (Tabela 01).

Os dados referentes à disponibilidade de fio/fita dental totalizam 11 marcas e 37 tipos de fio/fita dental. A disponibilidade foi de 17 tipos em 2004, passando para 34 em 2005 e 28 em 2006. Em 2004, a quantidade média de tipos de fio/fita dental encontrada entre as lojas do centro e as lojas dos bairros foi a mesma. No ano de 2005, assim como no ano de 2006, houve um pequeno aumento na disponibilidade destes produtos nas lojas centrais em relação às lojas situadas em outros bairros na proporção de 12/11, em 2005, e 11/9, em 2006.

A tabela 02 apresenta os dados dos produtos agrupados por ano e os valores encontrados. Observou-se que o preço da escova mais barata aumentou cerca de $\mathrm{R} \$ 0,10$ ao ano e o da escova mais cara aumentou $R \$ 2,06$ ao longo de 3 anos. Na média, o valor de todas as marcas e tipos de escovas encontradas aumentaram $R \$$ 1,00. Uma tendência decrescente no custo mínimo dos dentifrícios foi observada. Contudo os dentifrícios que apresentavam os preços mais altos mantiveram os mesmos valores. Em relação ao custo do fio/fita dental, o custo mínimo, em geral, sofreu um discreto aumento, enquanto o custo máximo sofreu uma discreta redução (Tabela 02).

Tabela 02. Valor das escovas dentais, dentifrícios e fio/fita dental encontrados nos supermercados do município de Cascavel, PR, entre 2004 e 2006.

\begin{tabular}{|c|c|c|c|}
\hline \multicolumn{4}{|c|}{ Escovas dentais } \\
\hline Preço & 2004 & 2005 & 2006 \\
\hline Mínimo & $\begin{array}{c}\mathrm{R} \$ 0,78 \\
\text { (Condor Plus) }\end{array}$ & $\begin{array}{c}\mathrm{R} \$ 0,89 \\
\text { (Ultra 2000) }\end{array}$ & $\begin{array}{c}\mathrm{R} \$ 0,99 \\
\text { (Condor Plus) }\end{array}$ \\
\hline Máximo & $\begin{array}{c}\mathrm{R} \$ 9,99 \\
\text { (Colgate Total } \\
\text { Professional) }\end{array}$ & $\begin{array}{c}\mathrm{R} \$ 12,00 \\
\text { (Oral-B Cross Action } \\
\text { Vitalizer) }\end{array}$ & $\begin{array}{c}\mathrm{R} \$ 12,05 \\
\text { (Oral-B Cross Action } \\
\text { Vitalizer) }\end{array}$ \\
\hline Médio & $R \$ 3,67$ & $\mathrm{R} \$ 4,59$ & $\mathrm{R} \$ 4,67$ \\
\hline \multicolumn{4}{|c|}{ Dentifrícios } \\
\hline Preço & 2004 & 2005 & 2006 \\
\hline Mínimo & $\begin{array}{c}\mathrm{R} \$ 0,85 \\
\text { (Free Dent - 90g) }\end{array}$ & $\begin{array}{c}\mathrm{R} \$ 0,74 \\
\text { (Ice Fresh }-50 \mathrm{~g} \text { ) }\end{array}$ & $\begin{array}{c}\mathrm{R} \$ 0,68 \\
\text { (Ice Fresh - } 50 \mathrm{~g} \text { ) }\end{array}$ \\
\hline Máximo & $\begin{array}{c}\mathrm{R} \$ 12,59 \\
\text { (Sensodyne Fresh Mint } \\
-50 \mathrm{~g})\end{array}$ & $\begin{array}{c}\mathrm{R} \$ 12,59 \\
\text { (Sensodyne Fresh Mint } \\
-50 \mathrm{~g})\end{array}$ & $\begin{array}{c}\mathrm{R} \$ 12,59 \\
\text { (Sensodyne } \\
\text { Branqueador }-50 \mathrm{~g} \text { ) }\end{array}$ \\
\hline Média & $R \$ 2,14$ & $R \$ 2,73$ & $R \$ 3,02$ \\
\hline \multicolumn{4}{|c|}{ Fio/fita dental } \\
\hline Preço & 2004 & 2005 & 2006 \\
\hline Mínimo & $\begin{array}{c}\mathrm{R} \$ 1,95 \\
\text { (Condor Medic } \\
\text { Tradicional - 25m) }\end{array}$ & $\begin{array}{c}\mathrm{R} \$ 1,75 \\
\text { (Hilo }-50 \mathrm{~m})\end{array}$ & $\begin{array}{c}\mathrm{R} \$ 1,98 \\
\text { (Hilo-50m) }\end{array}$ \\
\hline Máximo & $\begin{array}{c}\mathrm{R} \$ 8,25 \\
\text { (Johnson \& Johnson } \\
\text { Antitártaro - 40m) }\end{array}$ & $\begin{array}{c}\mathrm{R} \$ 9,04 \\
\text { (Johnson \& Johnson } \\
\text { Magic Grip - 40m) }\end{array}$ & $\begin{array}{c}\mathrm{R} \$ 7,65 \\
\text { (Johnson \& Johnson } \\
\text { Antitártaro }-40 \mathrm{~m} \text { ) }\end{array}$ \\
\hline Média & $\mathrm{R} \$ 4,01$ & $\mathrm{R} \$ 4,31$ & $\mathrm{R} \$ 4,25$ \\
\hline
\end{tabular}

Tabela 01. Disponibilidade de escovas dentais, dentifrícios e fio/fita dental nos supermercados do município de Cascavel, PR, entre 2004 e 2006.

\begin{tabular}{|c|c|c|c|c|c|c|c|c|c|c|c|}
\hline \multirow{2}{*}{ Lojas } & \multirow{2}{*}{ Rede de supermercado } & \multirow{2}{*}{ Localização } & \multicolumn{3}{|c|}{ Escovas dentais } & \multicolumn{3}{|c|}{ Dentifrícios } & \multicolumn{3}{|c|}{ Fio dental } \\
\hline & & & 2004 & 2005 & 2006 & 2004 & 2005 & 2006 & 2004 & 2005 & 2006 \\
\hline Loja 01 & Rede A & Bairros & 26 & 38 & 41 & 49 & 53 & 53 & 09 & 10 & 09 \\
\hline Loja 02 & Rede A & Centro & 27 & 47 & 55 & 49 & 53 & 49 & 09 & 14 & 16 \\
\hline Loja 03 & Rede A & Centro & 27 & 45 & 55 & 49 & 52 & 48 & 09 & 14 & 16 \\
\hline Loja 04 & Rede B & Centro & 15 & 30 & 44 & 35 & 53 & 51 & 07 & 10 & 11 \\
\hline Loja 05 & Rede B & Centro & 11 & 32 & 25 & 35 & 47 & 48 & 07 & 08 & 05 \\
\hline Loja 06 & Rede B & Bairros & 16 & 41 & 30 & 35 & 47 & 42 & 03 & 12 & 10 \\
\hline Loja 07 & Rede B & Centro & 20 & 42 & 48 & 35 & 47 & 45 & 03 & 13 & 09 \\
\hline Loja 08 & Rede C & Bairros & 22 & 35 & 30 & 44 & 40 & 43 & 06 & 09 & 08 \\
\hline Loja 09 & Rede C & Bairros & 22 & 35 & 30 & 44 & 40 & 43 & 06 & 09 & 08 \\
\hline Loja 10 & Rede C & Bairros & 22 & 35 & 30 & 44 & 40 & 43 & 06 & 09 & 08 \\
\hline Loja 11 & Rede D & Bairros & 25 & 37 & 38 & 45 & 57 & 51 & 10 & 16 & 09 \\
\hline \multirow[t]{4}{*}{ Loja 12} & Rede D & Bairros & 23 & 38 & 37 & 45 & 49 & 54 & 10 & 14 & 09 \\
\hline & & tal (Centro/Bairros) & $100 / 156$ & $196 / 259$ & $227 / 236$ & $203 / 306$ & $252 / 326$ & $241 / 329$ & $35 / 50$ & $59 / 79$ & $57 / 61$ \\
\hline & & dia (Centro/Bairros) & $20 / 22$ & $39 / 37$ & $45 / 34$ & $41 / 44$ & $50 / 47$ & $48 / 47$ & $7 / 7$ & $12 / 11$ & $11 / 9$ \\
\hline & Desvio $\mathrm{Pa}$ & ão (Centro/Bairros) & $7,1 / 3,2$ & $7,7 / 2,2$ & $12,3 / 4,8$ & $7,7 / 4,2$ & $3,1 / 6,9$ & $2,2 / 5,4$ & $2,4 / 2,6$ & $2,7 / 2,8$ & $4,7 / 0,8$ \\
\hline
\end{tabular}




\section{Discussão}

Verificou-se que a disponibilidade dos tipos de produtos comercializados aumentou consideravelmente em boa parte dos supermercados estudados no período em questão. Em 2004, por exemplo, nas lojas 02 e 03, eram comercializados 27 tipos de escovas enquanto que, em 2006, 55 tipos, ou seja, o dobro. De uma forma geral, houve um aumento de aproximadamente $56 \%$ na disponibilidade de escovas dentais nas lojas pertencentes às redes de supermercados pesquisadas entre os anos de 2004 e 2006.

Em estudo realizado no estado de Santa Catarina, 64\% dos entrevistados citaram o fator proximidade de casa e $20 \%$ como fator de conveniência para a escolha do supermercado onde faz suas compras. Caso houvesse a mesma tendência no presente estudo, a população situada próxima aos supermercados 02 ou 03 teria acesso a 55 tipos de escova dental para escolha, ou seja, mais que o dobro comparado com a população próxima ao supermercado 05, que tinha 25 tipos para escolha (BORGES, 2001).

Outro fator a ser considerado refere-se a falsa hipótese de que todas as lojas de uma mesma rede de supermercados tendem a apresentar a mesma quantidade para uma mesma mercadoria. Quando tratou-se de produtos como escova dental, verificou-se que isso não aconteceu, pois as lojas, em sua maioria, apresentaram divergência quanto a quantidade de tipos de produtos comercializados. Verificou-se, por exemolo, que nas lojas pertencentes a rede $B$, no ano de 2004, foram encontrados 11 tipos em uma loja e 20 em outra; em 2005, foram 32 e 42 tipos e, em 2006, 25 e 48 tipos de escovas dentais.

Sem dúvida, o custo da escova é um critério que muitos levam em consideração no momento de sua escolha, e que pode ter um peso maior em famílias com menor poder aquisitivo. No estudo de Lopes e Nascimento (1993), 60\% dos entrevistados que residiam na periferia, com renda familiar inferior a um salário mínimo escolhiam suas escovas pelo preço, ou seja, as que apresentavam menor custo.

A variabilidade de preço das escovas dentais no município de Cascavel, PR, observada no presente estudo, foi maior, em todos os períodos de avaliação (Tabela 02), que a encontrada em estudo realizado no município de Belo Horizonte, MG, no período compreendido entre setembro de 2005 e julho de 2006 (FEITOSA et al., 2008). Enquanto nele, o preço das escovas variou entre $\mathrm{R} \$$ 1,00 até $R \$ 8,55$, no presente estudo variou entre $R \$ 0,78$ a 12,05. Para o ano de 2006, o preço mínimo de uma escova dental $(\mathrm{R} \$ 0,99)$ foi praticamente o mesmo encontrado no estudo de Feitosa et al, $(R \$ 1,00)$, enquanto o preço médio $(R \$ 4,67)$ foi inferior ao encontrado no estudo dos mesmos autores (R\$4,99). Ainda, comparando os dois estudos, verificou-se que o preço máximo de uma escova dental comercializada no município de Cascavel, $\mathrm{PR},(\mathrm{R} \$ 12,05)$ era maior do que em Belo Horizonte, $M G$, $(R \$ 8,55)$.

Em relação aos dentifrícios, observou-se um aumento de aproximadamente $39,65 \%$ na disponibilidade destes produtos nos supermercados pesquisados durante o período em questão.

Segundo a empresa de marketing ACNielsen, em 2003, as três principais marcas de dentifrício concentravam $80 \%$ do mercado (ACNIELSEN, 2001). No fim de 2004, o mesmo instituto informava que havia 15 indústrias no país, produtoras de 23 marcas e 112 versões e tipos de dentifrícios (EMBALAGEMMARCA, 2004). Esse aumento no número de marcas e tipos de dentifrícios também foi observado no período em que foi realizada a presente pesquisa.

Quando se considerou o valor e o peso líquido, observouse que os dentifrícios de maior custo eram aproximadamente 18 vezes mais caros que os dentifrícios mais baratos. No entanto, o custo máximo desses dentifrícios não sofreu variação durante o período da pesquisa.

Já em relação aos dentifrícios de menor valor, os mesmos tornaram-se mais baratos durante o transcorrer da pesquisa, passando de $R \$$ 0,85 em 2004 para $R$ \$ 0,68 em 2006. Uma hipótese para tal fato pode ser devido ao aumento de $39,65 \%$ na disponibilidade de marcas e tipos de dentifrícios no período em questão, que pode ter contribuído para o aumento da concorrência e, como consequência, uma diminuição nos valores dos produtos mais populares e estabilização dos valores dos produtos mais onerosos.

Quando da avaliação da disponibilidade de fio/fita dental, observou-se um aumento de aproximadamente $64,71 \%$, porém, 9 diferentes tipos de fio dental foram retirados dos mercados e não encontrados no ano de 2006.

Infelizmente, o acesso e a utilização do fio dental no Brasil ainda são restritos às camadas altas e médias da sociedade. Dados de pesquisa realizada em 1996 indicaram que o brasileiro consumia uma média de $4 \mathrm{~cm}$ de fio dental ao ano (ANDRADE,1996).

Apesar da quantidade de fio/fita dental disponível no mercado ter praticamente dobrado nos anos de 2005 e 2006, manteve-se sempre muito mais baixo do que os tipos e marcas de escovas e dentrícios produzidos.

Quanto aos valores do fio/fita dental, apesar do custo médio ter sido de $\mathrm{R} \$ 4,15$, destaca-se que houve uma grande variação entre os valores encontrados, observando-se uma diferença de até 5 vezes entre um produto e outro.

Paralelamente ao fator custo dos produtos, o cirurgiãodentista deve ter em mente que o aumento da utilização de produtos de higiene bucal passa inevitavelmente pela conscientização da população quanto à importância do uso destes insumos para melhorar a qualidade de sua saúde bucal (ANDRADE, 1996).

\section{Conclusão}

Verificou-se uma grande quantidade de marcas e tipos de produtos para higienização bucal comercializados nas redes de supermercados avaliadas, porém, variações foram encontradas entre os anos de 2004 a 2006 entre as redes de supermercados, e dentre as lojas das redes de supermercados. Observou-se ainda, uma grande diferença entre os preços dos produtos mais caros e mais baratos, sendo essa maior entre as escovas dentais e dentifrícios.

A partir dos dados levantados, percebe-se a necessidade de os profissionais da área odontológica avaliarem esses aspectos quando recomendarem a aquisição desses produtos para os usuários por eles atendidos.

\section{Referências}

ACNIELSEN. Alcançando a Marca de um Bilhão de Dólares: Panorama das Marcas Globais Atuais. ACNielsen. São Paulo, 2001. Disponível em http://www.acnielsen.com.br. Acesso em 26 de ago. 2006.

ALBANDAR, J. M. Global Risk Factors and Risk Indicators for Periodontal Diseases. Periodontol. 2000. Birmingham. v.29, no. 2, p. 177-206, July 2002.

AMERICAN DENTAL ASSOCIATION. Cleaning your Teeth and Guns (Oral Hygiene). Disponível em www.ada.org/public/topics/cleaning faq.asp\#4. Acesso em 12 de out. 2006.

ANDRADE, M. O Nó do Mercado. Rev. ABO Nac. São Paulo. v.4. n.1, p.14-18, fev./mar. 1996.

AXELSSON, P.; LINDHE, J. Effect of Controlled Oral Hygiene Procedures on Caries and Periodontal Disease in Adults. Result After 6 Years. J. Clin. Periodontol. Copenhagen. v.8, no.2, p. 239-248, June 1981. 
BAUROTH, K. et al. The Efficacy of an Essencial oil Antiseptic Mouthrinse vs. Dental Floss in Controlling Interproximal Gingivitis: a Comparative Study. J. Am. Dent. Assoc. Chicago. v.134. no.3, p.359-365, Mar. 2003.

BORGES, A. D. Marketing de Varejo: as Estratégias Adotadas pelos Supermercados de Vizinhança para Conquistar e Fidelizar Clientes. 2001. 124p. Dissertação (Mestrado em Engenharia de Produção)

- Faculdade de Engenharia de Produção, Universidade Federal de Santa Catarina, Florianópolis, 2001.

CARVALHO, A. A. et al. O Custo Social da Higiene Oral na Cidade de Salvador. Rev. Fac. Odontol. Univ. Fed. Bahia, Salvador, v.7, n.1, p. 75-85, jan./dez. 1987.

CURY, J. A. Dentifrícios: Como Escolher e Como Indicar. In: CARDOSO, R. J. A.; GONÇALVES, E. A. N. Odontopediatria/Prevenção. São Paulo: Artes Médicas, 2002. p. 281-295 [Cap. 16].

EMBALAGEMMARCAS. Cremes Dentais: Cenário Sorridente, um Convite para Inovações. EmbalagemMarcas. São Paulo. n.63, nov. 2004. Disponível em http://www.embalagemmarca.com.br. Acesso em 16 de set. 2006.

FEITOSA, N.B. et al. Avaliação da Apresentação Comercial de Escovas Dentais Disponíveis no Brasil. Rev. Odonto Ciênc. Porto Alegre. v. 23, n.1, p. 77-81, jan./mar. 2008.

LOPES, W. C.; NASCIMENTO, Z. C. P. Avaliação da Preferência, Uso e Substituição de Escovas Dentais. ROBRAC. Goiânia. v. 2. n. 9, p. 4-10, dez. 1993.

O ESTADO DE SÃO PAULO. Marca própria ganha mais espaço na estratégia do varejo. Caderno de Economia \& Negócios. 29 de maio de 2010.

PERRY, D. A. Controle de Placa para o Paciente Periodontal. In: CARRANZA JUNIOR, F. A.; NEWMAN, M. G. Periodontia Clínica. 9 ed. Rio de Janeiro: Guanabara Koogan, p. 578-99, 2004 [Cap. 49].

SANTOS, L.F. et al. Custo da Higienização Bucal no Município de Cascavel,PR.RFOUPF.PassoFundo.v.13.n.2,p.12-16, maio/ago.2008.

TODESCAN, J. H. Usos e Costumes da Higiene Bucal - II. Rev. Assoc. Paul. Cir. Dent. São Paulo. v.45, n.5, p. 593-596, set./out. 1991.

VAN DER WEIJDEN, G. A.; HIOE, K. P. K. A Systematic Review of the Effectiveness of Self-performed Mechanical Plaque Removal in Adults with Gengivitis Using a Manual Toothbrush. J. Clin. Periodontol. Copenhagen, v.32, p. 214-228, 2005 [Suppl. 6]. 